\title{
Antithrombotic therapy in patients undergoing TAVI: an overview of Dutch hospitals
}

\author{
V. J. Nijenhuis • P. R. Stella • J. Baan • B. R. G. Brueren • P. P. de Jaegere • P. den Heijer • \\ S. H. Hofma - P. Kievit • T. Slagboom • A. F. M. van den Heuvel • F. van der Kley • \\ L. van Garsse • K. G. van Houwelingen • A. W. J. van't Hof • J. M. ten Berg
}

Published online: 28 November 2013

(C) The Author(s) 2013. This article is published with open access at Springerlink.com

\begin{abstract}
Purpose To assess current antithrombotic treatment strategies in the Netherlands in patients undergoing transcatheter aortic valve implantation (TAVI).

Methods For every Dutch hospital performing TAVI $(n=14)$ an interventional cardiologist experienced in performing TAVI was interviewed concerning heparin, aspirin, thienopyridine and oral anticoagulation treatment in patients undergoing TAVI.
\end{abstract}

Electronic supplementary material The online version of this article (doi:10.1007/s12471-013-0496-6) contains supplementary material, which is available to authorized users.

V. J. Nijenhuis $(\bowtie) \cdot J$. M. ten Berg

Department of Cardiology, St. Antonius Hospital, Koekoekslaan 1, 3435 Nieuwegein, the Netherlands

e-mail: v.nijenhuis@antoniusziekenhuis.nl

\section{P. R. Stella}

Department of Cardiology, University Medical Centre, Utrecht, the Netherlands

J. Baan

Department of Cardiology, Academic Medical Centre, Amsterdam, the Netherlands

\section{B. R. G. Brueren}

Department of Cardiology, Catharina Hospital, Eindhoven,

the Netherlands

P. P. de Jaegere

Department of Cardiology, Erasmus Medical Centre, Rotterdam, the Netherlands

P. den Heijer

Department of Cardiology, Amphia Hospital, Breda, the Netherlands

S. H. Hofma

Department of Cardiology, Medical Centre, Leeuwarden,

the Netherlands
Results The response rate was $100 \%$. In every centre, a protocol for antithrombotic treatment after TAVI was available. Aspirin was prescribed in all centres, concomitant clopidogrel was prescribed 13 of the 14 centres. Duration of concomitant clopidogrel was 3 months in over twothirds of cases. In 2 centres, duration of concomitant clopidogrel was based upon type of prosthesis: 6 months versus 3 months for supra-annular and intra-annular prostheses, respectively.

\section{P. Kievit}

Department of Cardiology, University Medical Centre St. Radboud, Nijmegen, the Netherlands

\section{T. Slagboom}

Department of Cardiology, Onze Lieve Vrouwe Gasthuis, Amsterdam, the Netherlands

\section{A. F. M. van den Heuvel}

Department of Cardiology, University Medical Centre, Groningen, the Netherlands

F. van der Kley

Department of Cardiology, University Medical Centre, Leiden, the Netherlands

L. van Garsse

Department of Cardiology, Medical University Centre, Maastricht, the Netherlands

\section{K. G. van Houwelingen}

Department of Cardiology, Medisch Spectrum Twente, Enschede, the Netherlands

A. W. J. van't Hof

Department of Cardiology, Isala Klinieken, Zwolle, the Netherlands 
Conclusions Leaning on a small basis of evidence and recommendations, the antithrombotic policy for patients undergoing TAVI is highly variable in the Netherlands. As a standardised regimen might further reduce haemorrhagic complications, large randomised clinical trials may help to establish the most appropriate approach.

Keywords Transcatheter Aortic Valve Implantation (TAVI) Clopidogrel · Aspirin · Dual Antiplatelet Therapy (DAPT) ·

Thrombosis $\cdot$ Bleeding

\section{Introduction}

Since its introduction in 2002, TAVI has conquered a substantial portion of aortic interventions. It has become standard therapy in inoperable patients [1]. Moreover, in high-risk but operable patients, TAVI has shown to be non-inferior to surgical aortic valve replacement [2]. Due to growing experience, smaller catheters, and new-generation devices, the rate of complications has been reduced. However, the incidence of bleeding and vascular complications remains substantial and has been proven to affect survival [3, 4].

A standardised antithrombotic treatment may reduce the rate of these negative outcomes. However, no large randomised controlled trials have been performed, and current guidelines report only recommendations [5-7]. The rationale for this survey was to provide insight into the current antithrombotic policy after TAVI. Results could be of value in decision-making on treatment policy for both experienced and inexperienced centres, and provide a basis for further research.

\section{Methods}

Setting and data collection

Currently, 14 hospitals in the Netherlands perform TAVI [8]. All centres were approached in the period from February to April 2013. In each hospital, we contacted the interventional cardiologist responsible for TAVI by an introduction e-mail addressing our intention to undertake a national survey. To maximise compliance, the interview was performed by visit $(n=4)$ or telephone $(n=9)$. In 1 case, communication was per e-mail. The interview was structured according to the printed survey depicted in the "Supplements".

\section{Assumptions}

In the development of the survey, we assumed antithrombotic treatment could be different per prosthesis or approach. We suspected it to be more dependent on the approach because of the bleeding risk, and designed the survey accordingly.
Besides closed questions for plain parameters such as dose and duration, we added two open questions for which we expected diverse answers.

Data analysis

Results are categorical and given as frequencies.

\section{Results}

Protocol

A local protocol for antithrombotic treatment in patients undergoing TAVI was available in all centres. In the majority of cases, the protocol was based upon local experience. Regarding the duration of dual antiplatelet therapy (DAPT), centres often followed the recommendations of the respective companies producing prostheses. These include 3 months of DAPT for Edwards SAPIEN ${ }^{\mathrm{TM}}$ (Edwards Lifesciences, Irvine, USA) and 6 months for Medtronic CoreValve ${ }^{\mathrm{TM}}$ (Medtronic, Minneapolis, USA).

\section{Procedure}

During the TAVI procedure, all centres used unfractionated heparin (UFH) as anticoagulant. The dose of UFH was according to weight in 3 centres and a standard initial bolus was given in 11 of the 14 centres, mostly $5000 \mathrm{IU}(n=6)$. Activated clotting time (ACT) was measured in 13 of the 14 centres. Target ACT was $>250 \mathrm{~s}(n=9),>200 \mathrm{~s}(n=2)$, or $>300 \mathrm{~s}(n=3)$.

In patients with an indication for oral anticoagulation (OAC), 3 centres continued OAC periprocedure with an international normalised ratio (INR) aimed at 2.0 or $<2.5$, whereas 11 of the 14 centres discontinued OAC periprocedure. Of these centres, 1 never performed bridging therapy, 3 always performed bridging therapy, and 7 performed bridging therapy in patients at high thrombotic risk only (e.g. mechanic mitral valve) as illustrated in Fig. 1.

\section{Concomitant antiplatelet therapy}

In patients without an indication for $\mathrm{OAC}$, all centres prescribed lifelong low-dose aspirin (80-100 mg). When a patient was not preloaded with aspirin, a loading dose (200$600 \mathrm{mg}$ ) was given in the week prior to procedure, mostly at day $-1(n=8)$. In addition to aspirin, 13 centres routinely prescribed clopidogrel as adjunctive antithrombotic medication. Clopidogrel was loaded in all centres with a loading dose of either $300(n=9)$ or $600 \mathrm{mg}(n=4)$. All centres prescribed a maintenance dose of $75 \mathrm{mg}$ for a varying duration of $1(n=1)$, $3(n=9)$, and $6(n=3)$ months (Fig. 2). 


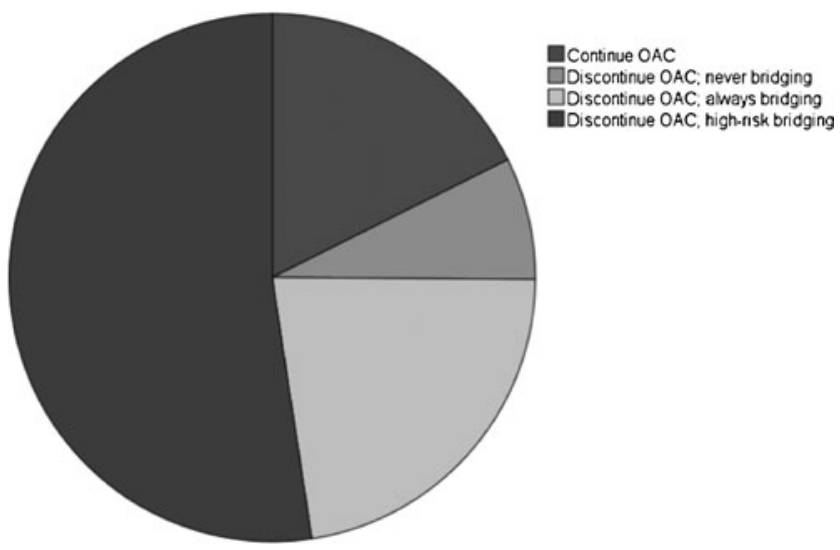

Fig. 1 Periprocedure antithrombotic policy in patients with an indication for oral anticoagulation (OAC)

One centre, which only performed transapical procedures, used no additional clopidogrel at all. In total, 4 centres discriminated in the addition of clopidogrel. In 2 centres, discrimination was based upon prosthesis type: SAPIEN ${ }^{\mathrm{TM}}$ versus CoreValve ${ }^{\mathrm{TM}}$. Whereas 1 centre reduced the loading dose (600 to $300 \mathrm{mg}$ ) and the duration (6 to 3 months) after SAPIEN ${ }^{\mathrm{TM}}$ implantation, the other centre omitted clopidogrel completely after SAPIEN ${ }^{\mathrm{TM}}$ implantation. In the other 2 centres, discrimination in DAPT was based upon approach: clopidogrel was loaded at day 0 for transfemoral TAVI and at day +1 for transapical TAVI.

In patients with an indication for OAC, concomitant antiplatelet therapy was used in 13 of the 14 centres for all prostheses and approaches. Concomitant antiplatelet therapy either consisted of clopidogrel $(n=9)$ or aspirin $(n=4)$ (Fig. 3). A single dose of aspirin in addition to clopidogrel at the time of loading was given in 1 centre. Two centres only used aspirin in addition to $\mathrm{OAC}$ when a patient was already loaded, otherwise clopidogrel was administered. Clopidogrel was loaded in all centres with a loading dose of either $300(n=5)$ or $600 \mathrm{mg}$

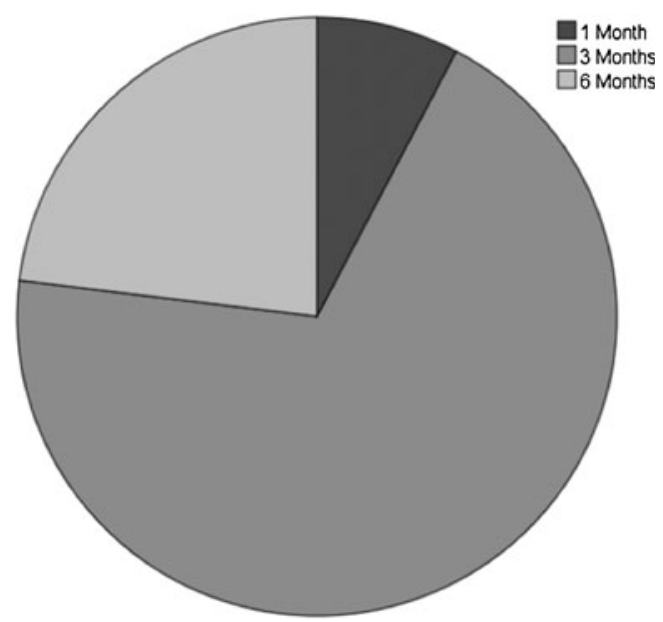

Fig. 2 Duration of dual antiplatelet therapy (DAPT) after TAVI in patients without an indication for oral anticoagulation (OAC)

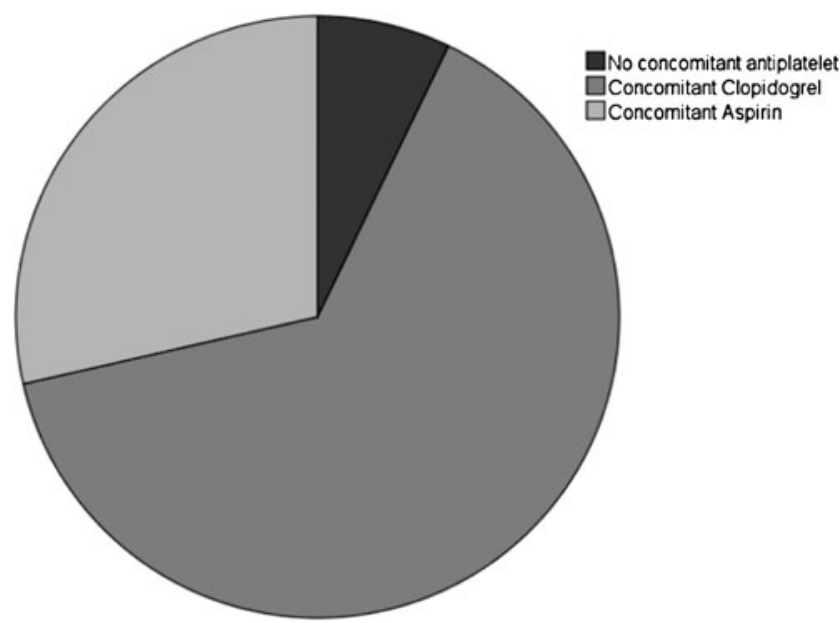

Fig. 3 Concomitant antiplatelet therapy after TAVI in patients with an indication for oral anticoagulation (OAC)

$(n=4)$. All centres prescribed a maintenance dose of $75 \mathrm{mg}$ for a varying duration of $1(n=1), 3(n=6)$, and $6(n=2)$ months. Considering aspirin, patients received a loading dose at day -1 $(n=1)$ or day $0(n=1)$ and a maintenance dose for 6 months $(n=1)$ or lifelong $(n=3)$.

In total, 2 of the 14 centres discriminated in the addition of clopidogrel. In both of these centres, this discrimination was based upon prosthesis type: SAPIEN ${ }^{\mathrm{TM}}$ versus CoreValve ${ }^{\mathrm{TM}}$. Whereas 1 centre reduced the loading dose (600 to $300 \mathrm{mg}$ ) and the duration (6 to 3 months) after SAPIEN ${ }^{\mathrm{TM}}$ implantation, the other centre omitted clopidogrel completely after SAPIEN $^{\mathrm{TM}}$ implantation. Centres prescribing aspirin did not discriminate between prosthesis types or approaches.

\section{Bleeding risk}

The prescribed antithrombotic regimen was based upon bleeding risk in 7 of the 14 centres. Three centres omitted clopidogrel completely in case of very high bleeding risk or severe thrombocytopenia according to local protocol. One centre reduced the duration of clopidogrel treatment (6 to 3 months) in case of a high bleeding risk. Two centres adjusted the time of commencing and ceasing clopidogrel treatment individually.

\section{DAPT pre-TAVI}

When a patient had undergone a recent ( $\leq 1$ year) PCI and had an absolute indication for DAPT prior to TAVI, all centres continued DAPT. When a patient received a newer thienopyridine (e.g. prasugrel, ticlopidine), the same therapy was continued in 8 centres whereas the thienopyridine was changed to clopidogrel in 2 of the 14 centres. The other 4 centres had no experience with such a scenario. 
Triple therapy pre-TAVI

If a patient was receiving triple therapy (i.e. OAC and DAPT) prior to TAVI, only 1 centre continued this. Triple therapy was routinely discontinued in 6 centres or continued for as short as possible (1-2 weeks) in 2 of the 14 centres. Two centres preferred not to continue triple therapy and adjusted medication on an individual basis, whereas 3 centres had no experience with such cases.

If triple therapy was discontinued, aspirin was omitted routinely in 4 centres and clopidogrel in 1 centre. Two centres omitted aspirin only when the patient received triple therapy because of a recent stent and omitted clopidogrel in other cases. OAC was omitted until clopidogrel could be stopped in 1 centre.

New-onset persistent atrial fibrillation

When a patient developed persistent atrial fibrillation (AF) after TAVI during the hospitalisation period and had an indication for OAC, aspirin was replaced by OAC in 9 of the 14 centres. Four centres only replaced aspirin by OAC when the patient received DAPT prior to TAVI because of a recent stent and replaced clopidogrel by OAC in other cases. OAC was added to DAPT in 1 centre.

\section{Discussion}

TAVI is an accepted treatment for high-risk patients with severe symptomatic AS and is the first choice of treatment for inoperable patients $[1,2]$. Nevertheless, TAVI remains associated with a relatively high number of haemorrhagic and embolic complications [3, 4]. Moreover, little is known about antithrombotic treatment strategies and current guidelines report only recommendations [5-7] In this survey, we interviewed centres performing TAVI in the Netherlands regarding antithrombotic treatment after TAVI. Although mostly according to one or more recommendations, inter-centre variability of the antithrombotic policy is high.

Haemorrhagic complications are frequent, and major and disabling or life-threatening bleeding after TAVI occurs in approximately $22 \%$ and $16 \%$, respectively [3]. Regardless of the mechanism, a disabling or life-threatening bleeding is an important predictor of acute and late mortality [9-13]. More insight into the role of the antithrombotic treatment regimen could provide the most desirable antithrombotic treatment to possibly reduce the rate of disabling or life-threatening bleeding and adverse outcomes [6].

The most prevalent thromboembolic complication is stroke, which occurs in approximately $2.5 \%$ to $4.1 \%$ at 30 days [4, 14-16]. Most acute strokes are procedure related and of embolic origin, caused by manipulation with large catheters [17]. The incidence of late stroke is approximately $2 \%$ and similar to that observed to other populations of elderly people $[11,18]$. Late strokes are more prevalent in patients with classical risk factors such as (new-onset) AF [17, 19]. However, in the PARTNER IB trial, the portion of haemorrhagic stroke after 30 days was higher in the TAVI group than in the medical treatment group [18], emphasising the double-edged sword of antithrombotic treatment in the elderly.

Only the American College of Cardiology Foundation/ Society of Thoracic Surgeons (ACCF/AATS/SCAI/STS) Expert Consensus Document on TAVI has mentioned antithrombotic treatment during procedure and recommends UFH with an ACT $>300 \mathrm{~s}$ [6]. In the PARTNER I trial, UFH was given in an initial bolus of $5000 \mathrm{IU}$ with a target ACT $>250 \mathrm{~s}$ [1, 2]. In this survey, we found UFH was used in all centres and ACT is routinely measured in 13 of the 14 interviewed centres. The target ACT is $>300$ in only 3 centres and $>250 \mathrm{~s}$ in 9 of the 14 centres.

Following TAVI, DAPT consisting of aspirin (lifelong) and clopidogrel (initial period) is currently recommended [5-7]. However, this is largely based on retrospective registries and 2 cases of severe postprocedural thrombocytopenia in patients treated with clopidogrel without a loading dose in a preliminary first-in-man study with the CoreValve ${ }^{\mathrm{TM}}$ [20]. A second rationale for adding clopidogrel to aspirin is supported by the histopathological finding that incorporation of the CoreValve ${ }^{\mathrm{TM}}$ prosthesis into the aortic wall by means of endothelialisation is a chronological integration over a period of approximately 3 months [21].

Whereas aspirin (lifelong) is recommended unanimously, recommendations for the duration of clopidogrel vary from 1 to 3 months [7], 1 to 6 months [5] and 3 to 6 months [6]. In this survey, we found aspirin was prescribed in all centres according to recommendations. However, concomitant clopidogrel was prescribed in 13 of the 14 centres. Duration of concomitant clopidogrel therapy was 3 months in over two-thirds of cases. In 2 centres, duration of concomitant clopidogrel was based upon type of prosthesis: 6 months for CoreValve ${ }^{\mathrm{TM}}$ versus 3 months for intra-annular prostheses (e.g. SAPIEN ${ }^{\mathrm{TM}}$, JenaValve ${ }^{\mathrm{TM}}$ ). These centres tended to be more careful with CoreValve ${ }^{\mathrm{TM}}$ because of the supra-annular design and longer struts, and possible subsequent proneness to more thromboembolic complications, although this has not been described in the literature [4].

In patients with $\mathrm{AF}$ or another indication for $\mathrm{OAC}$, the best antithrombotic treatment regimen is unknown. In these patients, a combination of OAC and aspirin or thienopyridine is generally used [5]. We found that in patients with an indication for OAC, 13 of the 14 centres prescribed concomitant antiplatelet therapy. Of these, 9 centres used clopidogrel and 4 centres used aspirin.

Concomitant antiplatelet drugs have shown to increase the risk for major bleeding in patients with $\mathrm{AF}$ [22-24]. A recent Danish registry study shows that in patients with $\mathrm{AF}$ and myocardial infarction, the combination of $\mathrm{OAC}+$ clopidogrel was comparable with triple therapy [25]. However, in the recent WOEST trial, OAC+clopidogrel was superior to triple 
therapy in patients with AF undergoing drug-eluting stent placement including a striking decrease in all-cause mortality [26]. In this survey, 1 centre would continue triple therapy when a patient received triple therapy prior to TAVI. When discontinued, 4 centres omitted aspirin in any case and 1 centre omitted clopidogrel in any case. Two centres omitted aspirin only when patients received triple therapy because of a recent stent and omitted clopidogrel in other cases. One centre omitted OAC until clopidogrel could be stopped.

Leaning on a small basis of evidence and recommendations, we found considerable heterogeneity in the antithrombotic treatment practised between TAVI performing centres in the Netherlands. In most TAVI registries, information about antithrombotic treatment is not available. Generalising national antithrombotic policy may homogenise this factor, making clinically important outcomes more comparable in addition to the initiative of VARC-2 [27]. Furthermore, further research is needed to study the effect of antithrombotic therapy on clinical outcomes. We are currently setting up a multicentre randomised controlled clinical trial in the Netherlands, investigating the safety and efficacy of omitting clopidogrel in the first 3 months after TAVI versus aspirin+clopidogrel in patients without an indication for $\mathrm{OAC}$ at baseline, and versus $\mathrm{OAC}+$ clopidogrel in patients with an indication for $\mathrm{OAC}$ at baseline.

\section{Study limitations}

This study presents a description of current clinical practice in Dutch hospitals with respect to antithrombotic therapy in patients undergoing TAVI. It does not describe the outcome and complications of these treatment methods and none of the interviews were verified with patient data. Furthermore, this study only describes the current antithrombotic strategy and does not consider possible previous antithrombotic strategies or the experience of centres with the TAVI procedure. Finally, we approached only one cardiologist per centre assuming that cardiologists belonging to a single hospital and organisation apply the same strategies.

\section{Conclusion}

Leaning on a small basis of evidence and recommendations, the antithrombotic policy for patients undergoing TAVI is highly variable in the Netherlands. As a standardised regimen might further reduce haemorrhagic complications, large randomised clinical trials may help to establish the most appropriate approach.

\section{Funding None.}

Conflict of interest None declared.
Open Access This article is distributed under the terms of the Creative Commons Attribution License which permits any use, distribution, and reproduction in any medium, provided the original author(s) and the source are credited.

\section{References}

1. Leon MB, Smith CR, Mack M, et al. Transcatheter aortic-valve implantation for aortic stenosis in patients who cannot undergo surgery. N Engl J Med. 2010;363(17):1597-607.

2. Smith CR, Leon MB, Mack MJ, et al. Transcatheter versus surgical aortic-valve replacement in high-risk patients. N Engl J Med. 2011;364(23):2187-98.

3. Généreux P, Head SJ, Van Mieghem NM, et al. Clinical outcomes after transcatheter aortic valve replacement using valve academic research consortium definitions: a weighted metaanalysis of 3,519 patients from 16 studies. J Am Coll Cardiol. 2012;59(25):2317-26.

4. Khatri PJ, Webb JG, Rodes-Cabau J, et al. Adverse effects associated with transcatheter aortic valve implantation a meta-analysis of contemporary studies. Ann Intern Med. 2013;158(1):35-46.

5. Vahanian A, Alfieri O, Andreotti F, et al. Guidelines on the management of valvular heart disease (version 2012). Eur Heart J. 2012;33(19):2451-96.

6. Holmes DR, Mack MJ, Kaul S, et al. 2012 ACCF/AATS/SCAI/STS expert consensus document on transcatheter aortic valve replacement. J Am Coll Cardiol. 2012;59(13):1200-54.

7. Webb J, Rodés-Cabau J, Fremes S, et al. Transcatheter aortic valve implantation: a Canadian Cardiovascular Society position statement. Can J Cardiol. 2012;28(5):520-8.

8. THV-Group. Dutch Guidelines for Transcatheter Heart Valve Intervention [Internet]. 2012. Available from: https://www.nvvc.nl/ media/richtlijn/167/Guidelines\%20THI\%202012-DEF.pdf.

9. Borz B, Durand E, Godin M, et al. Incidence, predictors and impact of bleeding after transcatheter aortic valve implantation using the balloon-expandable Edwards prosthesis. Heart. 2012; 303095:1-6.

10. Halliday BP, Dworakowski R, Brickham B, et al. Usefulness of periprocedural bleeding to predict outcome after transcatheter aortic valve implantation. Am J Cardiol. 2012;109(5):724-8.

11. Kodali SK, Williams MR, Smith CR, et al. Two-year outcomes after transcatheter or surgical aortic-valve replacement. N Engl J Med. 2012;366(18):1686-95.

12. Van Mieghem NM, Nuis R-J, Tzikas A, et al. Prevalence and prognostic implications of baseline anaemia in patients undergoing transcatheter aortic valve implantation. EuroIntervention. 2011;7(2):184-91.

13. Ussia GP, Barbanti M, Petronio AS, et al. Transcatheter aortic valve implantation: 3-year outcomes of self-expanding CoreValve prosthesis. Eur Heart J. 2012;33(8):969-76.

14. Gilard M, Eltchaninoff H, Iung B, et al. Registry of transcatheter aortic-valve implantation in high-risk patients. N Engl J Med. 2012;366(18):1705-15.

15. Moat NE, Ludman P, De Belder M, et al. Long-term outcomes after transcatheter aortic valve implantation in high-risk patients with severe aortic stenosis: the U.K. TAVI (United Kingdom Transcatheter Aortic Valve Implantation) Registry. J Am Coll Cardiol. 2011;58(20):2130-8.

16. Zahn R, Gerckens U, Grube E, et al. Transcatheter aortic valve implantation: first results from a multi-centre real-world registry. Eur Heart J. 2011;32(2):198-204.

17. Nombela-Franco L, Webb JG, De Jaegere PP, et al. Timing, predictive factors, and prognostic value of cerebrovascular events in a large 
cohort of patients undergoing transcatheter aortic valve implantation. Circulation. 2012;126(25):3041-53.

18. Makkar RR, Fontana GP, Jilaihawi H, et al. Transcatheter aorticvalve replacement for inoperable severe aortic stenosis. N Engl J Med. 2012;366(18):1696-704.

19. Nuis R-J, Van Mieghem NM, Schultz CJ, et al. Frequency and causes of stroke during or after transcatheter aortic valve implantation. Am J Cardiol. 2012;109(11):1637-43.

20. Grube E, Laborde JC, Gerckens U, et al. Percutaneous implantation of the CoreValve self-expanding valve prosthesis in high-risk patients with aortic valve disease: the Siegburg first-in-man study. Circulation. 2006;114(15):1616-24.

21. Noble S, Asgar A, Cartier R, et al. Anatomo-pathological analysis after CoreValve Revalving system implantation. EuroIntervention. 2009;5(1):78-85.

22. Dans AL, Connolly SJ, Wallentin L, et al. Concomitant use of antiplatelet therapy with dabigatran or warfarin in the Randomized Evaluation of Long-Term Anticoagulation Therapy (RE-LY) trial. Circulation. 2013;127(5):634-40.
23. Hansen ML, Sørensen R, Clausen MT, et al. Risk of bleeding with single, dual, or triple therapy with warfarin, aspirin, and clopidogrel in patients with atrial fibrillation. Arch Intern Med. 2010;170(16): $1433-41$.

24. Karjalainen PP, Porela P, Ylitalo A, et al. Safety and efficacy of combined antiplatelet-warfarin therapy after coronary stenting. Eur Heart J. 2007;28(6):726-32.

25. Lamberts M, Gislason GH, Olesen JB, et al. Oral anticoagulation and antiplatelets in atrial fibrillation patients after myocardial infarction and coronary intervention. J Am Coll Cardiol. 2013 Jun 6.

26. Dewilde WJ, Oirbans T, Verheugt FW, et al. Use of clopidogrel with or without aspirin in patients taking oral anticoagulant therapy and undergoing percutaneous coronary intervention: an open-label, randomised, controlled trial. Lancet. 2013 Feb;12: 1107-15.

27. Kappetein AP, Head SJ, Généreux P, et al. Updated standardized endpoint definitions for transcatheter aortic valve implantation: the Valve Academic Research Consortium-2 consensus document. J Am Coll Cardiol. 2012;60(15):1438-54. Elsevier Inc. 